Marquette University

e-Publications@Marquette

9-4-2018

\title{
Direct Observation of Node-to-Node Communication in Zeolitic Imidazolate Frameworks
}

\author{
Brian Pattengale \\ Marquette University \\ Daniel J. SantaLucia \\ University of Wisconsin - Madison \\ Sizhuo Yang \\ Marquette University \\ Wenhui Hu \\ Marquette University \\ Cunming Liu \\ Argonne National Laboratory
}

See next page for additional authors

Follow this and additional works at: https://epublications.marquette.edu/chem_fac

Part of the Chemistry Commons

\section{Recommended Citation}

Pattengale, Brian; SantaLucia, Daniel J.; Yang, Sizhuo; Hu, Wenhui; Liu, Cunming; Zhang, Xiaoyi; Berry, John F.; and Huang, Jier, "Direct Observation of Node-to-Node Communication in Zeolitic Imidazolate

Frameworks" (2018). Chemistry Faculty Research and Publications. 973.

https://epublications.marquette.edu/chem_fac/973 


\section{Authors}

Brian Pattengale, Daniel J. SantaLucia, Sizhuo Yang, Wenhui Hu, Cunming Liu, Xiaoyi Zhang, John F. Berry, and Jier Huang

This article is available at e-Publications@Marquette: https://epublications.marquette.edu/chem_fac/973 
Marquette University

e-Publications@Marquette

\section{Chemistry Faculty Research and Publications/College of Arts and Sciences}

This paper is NOT THE PUBLISHED VERSION; but the author's final, peer-reviewed manuscript. The published version may be accessed by following the link in th citation below.

Journal of the American Chemical Society, Vol. 140, No. 37 (September 4, 2018): 11573-11576. DOI. This article is (C) American Chemical Society and permission has been granted for this version to appear in e-Publications@Marquette. American Chemical Society does not grant permission for this article to be further copied/distributed or hosted elsewhere without the express permission from American Chemical Society.

\section{Direct Observation of Node-to-Node Communication in Zeolitic Imidazolate Frameworks}

Brian Pattengale

Department of Chemistry, Marquette University, Milwaukee, Wisconsin Daniel J. SantaLucia

Department of Chemistry, University of Wisconsin, Madison, Wisconsin Sizhuo Yang Department of Chemistry, Marquette University, Milwaukee, Wisconsin Wenhui Hu

Department of Chemistry, Marquette University, Milwaukee, Wisconsin Cunming Liu X-ray Science Division, Argonne National Laboratory, Argonne, Illinois Xiaoyi Zhang X-ray Science Division, Argonne National Laboratory, Argonne, Illinois John F. Berry Department of Chemistry, University of Wisconsin, Madison, Wisconsin 


\section{Jier Huang}

Department of Chemistry, Marquette University, Milwaukee, Wisconsin

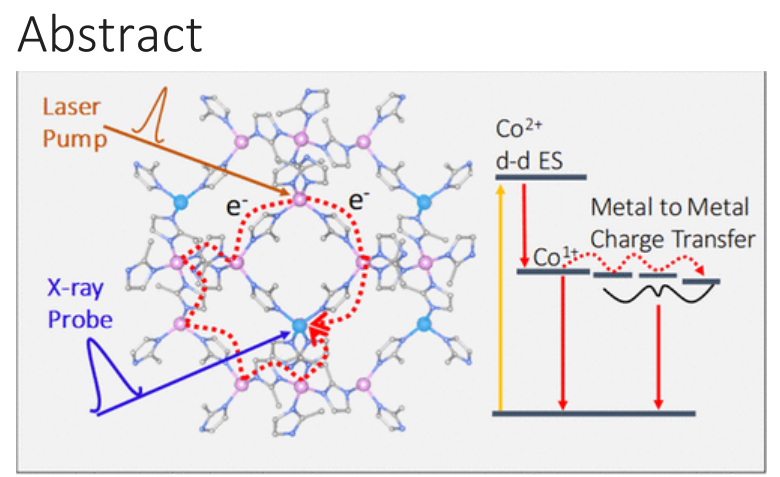

Zeolitic imidazolate frameworks (ZIFs) with open-shell transition metal nodes represent a promising class of highly ordered light harvesting antennas for photoenergy applications. However, their charge transport properties within the framework, the key criterion to achieve efficient photoenergy conversion, are not yet explored. Herein, we report the first direct evidence of a charge transport pathway through node-to-node communication in both ground state and excited state ZIFs using the combination of paramagnetic susceptibility measurements and time-resolved optical and X-ray absorption spectroscopy. These findings provide unprecedented new insights into the photoactivity and charge transport nature of ZIF frameworks, paving the way for their novel application as light harvesting arrays in diverse photoenergy conversion devices.

In natural photosynthesis, a highly ordered architecture composed of light-harvesting pigments plays the key role of light absorption and subsequent energy migration to the reaction centers.(1) Inspired from nature, an artificial photocatalytic system to generate solar fuels requires the utility of ordered chromophore assemblies as efficient light harvesting antennas. Metal-organic frameworks (MOFs), an emerging class of porous crystalline materials that can precisely arrange chromophores through ordered ligand-to-metal coordination, represent excellent platforms as light harvesting antennas.(2-15) A particularly interesting subclass of such light harvesting MOFs are zeolitic imidazolate frameworks (ZIFs),(16-18) which were recently identified to have intrinsic photophysical properties with broad absorption across UV-visible and near-IR spectral regions and an exceptionally long-lived excited state (2.9 $\mu$ s lifetime).(19-21) More interestingly, this long-lived excited state was found to be an excited charge separated (ECS) state that can be dissociated through electron transfer to an organic species,(22) showing enormous promise toward the application of ZIFs as next generation light harvesting and energy delivery antennas for solar energy conversion.

A judicious question that emerged following the discovery of the long-lived ECS state is whether this state can undergo facile charge transport across a ZIF framework, a key criterion to achieve efficient photoenergy conversion. Recent theoretical studies reported the electronic structure of ZIFs; the $\mathrm{d}$ orbitals of the metal nodes can be conceived as comprising a continuous band,(23-26) in agreement with the improved conductivity in $\mathrm{Co}^{2+}-$ based ZIFs with respect to highly resistive closed-shell Zn²+-based ZIFs.(27) While such exploratory work implies the possibility of charge transport via metal nodes in ZIFs, it is essential to establish an original approach to seek direct evidence of the charge transport mechanism experimentally. 
Herein, we uncover the nature of charge transport in ZIF materials using advanced spectroscopic methods. We designed mixed-metal isostructural ZIFs ( (which has $\mathrm{Co}^{2+}$ at the metal nodes connected by 2-methyl imidazolate linkers). We hypothesized that a guest metal would perturb the ECS dynamics if charge transport occurs within the ZIF framework; redox-inert metals (such as $\mathrm{Zn}^{2+}$ ) incorporated into the framework may prevent charge transport while redox-active guest metals (such as $\mathrm{Cu}^{2+}$ ) may facilitate charge transport through metal-to-metal charge transfer (MMCT) pathways. Using the combination of paramagnetic susceptibility measurements (obtained via a SQUID), optical transient absorption (OTA), and X-ray transient absorption (XTA) spectroscopy to directly probe the interplay between the guest and parent metals within the parent framework, we not only show the first-ever reported intrinsic antiferromagnetic ordering of metal nodes in ground state ZIFs but also demonstrate direct evidence of charge transport through node-to-node communication in photoexcited ZIFs.

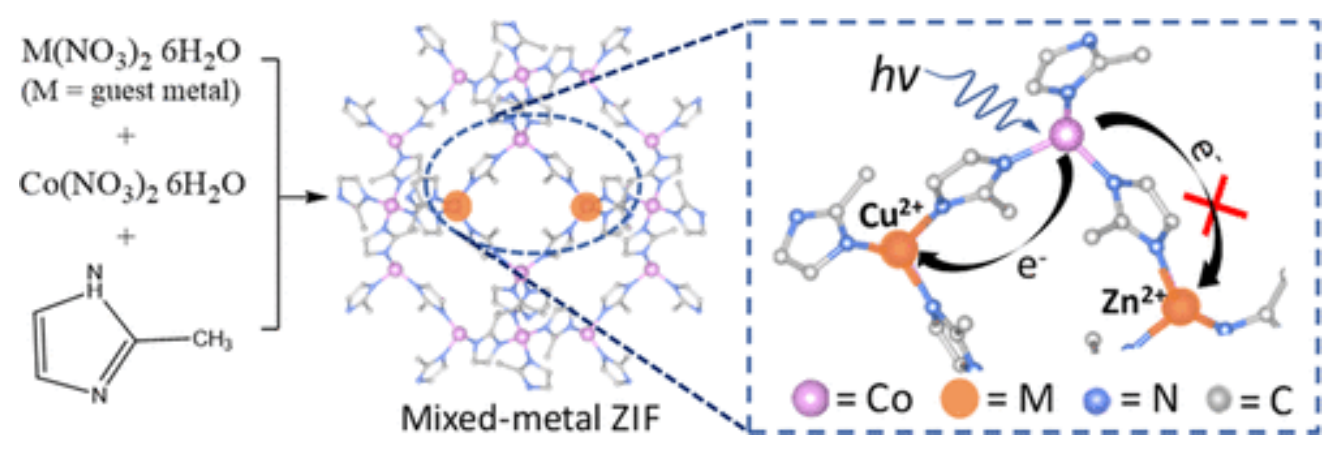

Scheme 1. Schematic Representation of Mixed-Metal ZIF-67

The mixed-metal ZIFs were synthesized by incorporating controlled amounts of $\mathrm{Zn}^{2+}$ salts and $\mathrm{Cu}^{2+}$ salts into $\mathrm{Co}^{2+}$ salts (X\%M@ZIF-67; X = the percent of guest metal; $\mathrm{M}=$ guest metal) in the presence of 2-methylimidazole (Scheme 1), in contrast to previously reported postsynthetic metal substation methods.(28-30) This innovative approach allowed the incorporation of a relatively larger content of guest metals in a controlled manner before the dissolution of the parent materials. The concentration of each metal node in the mixed-metal ZIFs was confirmed by ICP-MS (Table S1). The XRD patterns and X-ray absorption spectra (XAS) at the Co K-edge of these mixed-metal ZIFs resemble that of the parent ZIFs (igires S1-S3 ), indicating that the incorporation of guest metals has a negligible effect on the crystal structure of the parent ZIFs. The coordination environment at the guest metal center was determined to be tetrahedral by XAS (Figures S4-S5). The tetrahedral environment at $\mathrm{Cu}^{2+}$ centers in Cu@ZIF-67 was further supported by its reflective UV-visible-NIR spectra (Figure S6), where two prominent absorption bands are observed at 400-580 nm and 600-1600 nm, corresponding to ligand-to-metal charge transfer and $\mathrm{Cu} d-d$ transitions in a tetrahedral ligand field, respectively.(31-34)

The ground state interaction among metal nodes in mixed-metal ZIFs was investigated by SQUID magnetometry. As shown in Figure 1a (representative data set for 15\%Cu@ZIF-67) and Figure S7, the paramagnetic susceptibility data for ZIF-67 and all Cu@ZIF-67 samples all exhibit a transition to an antiferromagnetically ordered state at temperatures lower than $21 \mathrm{~K}\left(T_{\mathrm{N}}\right)$. Below $T_{\mathrm{N}}$, thermal energy is insufficient to disrupt the longrange order that causes all neighboring spins to cancel each other,(35) which is antiferromagnetic behavior. Figure $1 b$ shows the plot of $\chi_{P} T$ vs $T$ and a Curie plot for $15 \% \mathrm{Cu} @ Z I F-67$. The observed curvature in the plot of $\chi_{\mathrm{P}} T$ vs $T$ above $T_{\mathrm{N}}$ is characteristic of the ${ }^{4} \mathrm{~A}_{2}$ state of tetrahedral $\mathrm{Co}^{2+}$. The antiferromagnetism of the ZIF samples is quantified by the Weiss constants, $\Theta(\underline{\mathrm{S} 1}$, Table $\mathbf{S} 2)$, derived from the $x$-intercepts of the Curie plots. The $\Theta$ values range from -29 to $-32 \mathrm{~K}$ regardless of the amount of $\mathrm{Cu}$ doping. Control experiments with $\mathrm{Zn}-$ doped ZIF-67 samples were also conducted (Figure S9). Comparing the data for ZIF-67, 15\%Zn@ZIF-67, and 
15\%Cu@ZIF-67 shows antiferromagnetic ordering only in the $100 \% \mathrm{Co}$ and $15 \% \mathrm{Cu}$ samples (Figure S8). Inclusion of $\mathrm{Zn}^{2+}$ shuts down the magnetic exchange interactions responsible for the ordering behavior, while inclusion of $\mathrm{Cu}^{2+}$ enforces the magnetic ordering.
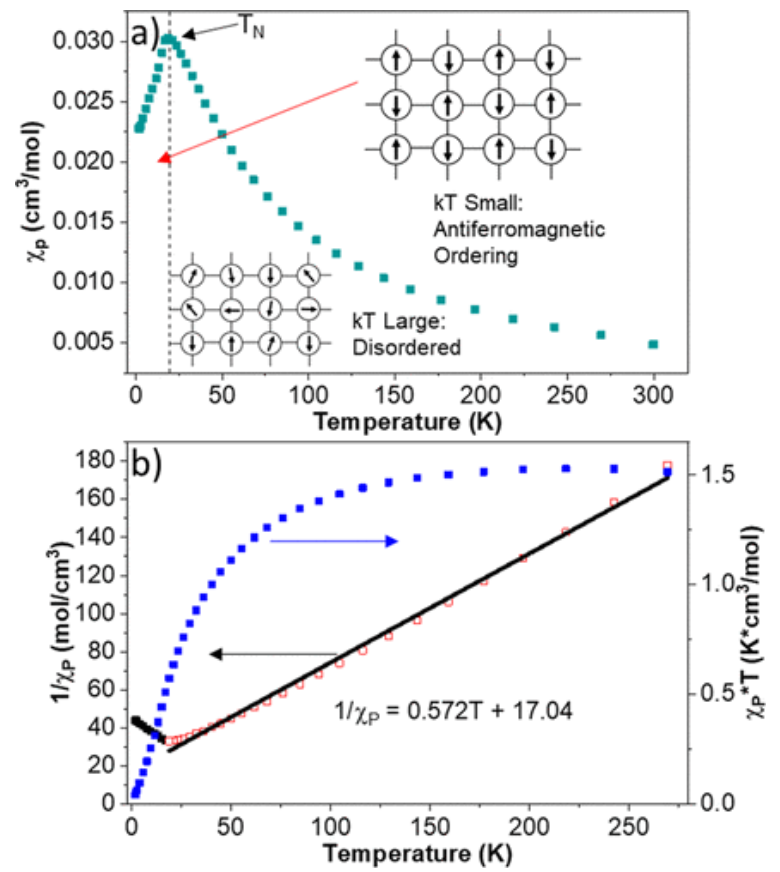

Figure 1. (a) Magnetic susceptibility data of 15\%Cu@ZIF-67. (b) Plot of $\chi_{P} T$ vs $T$ (blue) and a Curie plot (red) for 15\%Cu@ZIF67. The least-squares fit (black) over the quasi-linear region of the Curie plot is superimposed on the red trace.

To examine the charge transport nature of ZIFs, we first investigated the effect of redox inert metals $\left(\mathrm{Zn}^{2+}\right)$ on ECS dynamics using OTA spectroscopy. Two ZIF model systems, 80\%Zn@ZIF-67 and 50\%Zn@ZIF-67, were studied. While these samples are isostructural with the ZIF-67 parent framework, the UV-visible-NIR spectra show decreasing absorption of both Co $d-d$ and LMCT transitions (Figure 2a) with increasing $\mathrm{Zn}^{2+}$ content due to the dilution of the $\mathrm{Co}^{2+}$ chromophores by the nonabsorbing $\mathrm{Zn}^{2+}$ with a $3 \mathrm{~d}(10)$ electron configuration. This is also reflected in their OTA spectra following the excitation of the lower lying $\mathrm{Co}^{2+} d-d$ transition $\left({ }^{4} A_{2}(F)-{ }^{4} T_{1}(F)\right)$ at $1000 \mathrm{~nm}$, where the intensity of OTA spectra decreases significantly with increasing content of $\mathrm{Zn}^{2+}$ (Figures $2 \mathrm{~b}$ and $\underline{\mathrm{S} 10}$ ). Nevertheless, the shape of the OTA spectra, featuring a ground state bleach (GSB) at $585 \mathrm{~nm}$ and positive absorption at both sides of the GSB remains similar among the mixed-metal ZIFs. These spectral signatures are consistent with the derivative feature observed previously in ZIF-67(20) and can thus be assigned to the formation of a long-lived ECS state in Zn@ZIF-67. Despite the formation of the ECS state in Zn@ZIF-67, significant differences were observed when we compared their kinetic traces, where the recovery of the GSB became faster (inset of Figure 2c) while the formation of the ECS state is slower in the films with higher $\mathrm{Zn}^{2+}$ content (Figure 2c). Because of the closed-shell configuration of $\mathrm{Zn}^{2+}$, it is unlikely that MMCT would occur from $\mathrm{Co}^{2+}$ to $\mathrm{Zn}^{2+}$. Instead, these results can be well explained by a model proposed in the inset of Figure 2a: the presence of a statistical number of $\mathrm{Zn}^{2+}$ neighbors with closed-shell configuration partially blocks the inherent ECS pathway in ZIF-67 through Co-Co communication, resulting in slower formation of the ECS state. As a result, direct transition from ${ }^{4} T_{1}$ to ${ }^{4} A_{2}$ is facilitated, which leads to faster GSB recovery kinetics. 

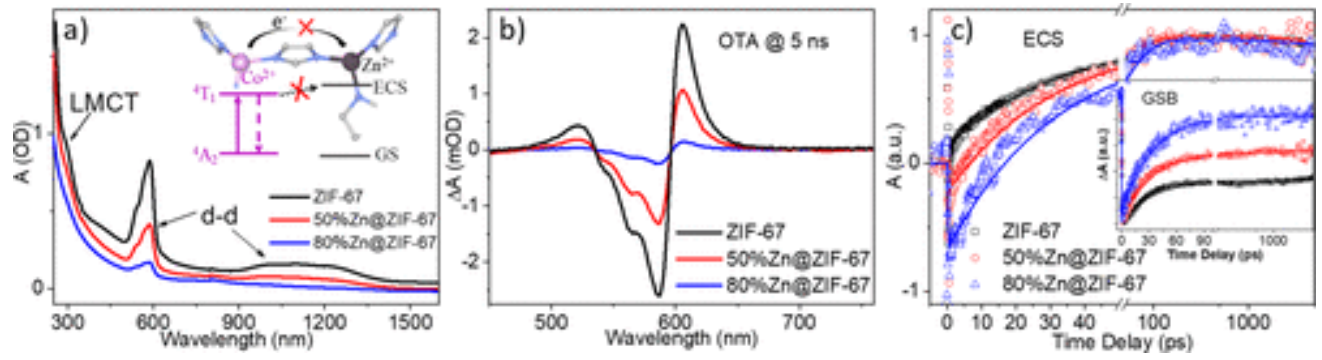

Figure 2. (a) UV-visible-NIR absorption spectra of $\mathrm{Zn}^{2+}$ incorporated ZIF-67 with different Zn percent (X\%Zn@ZIF-67). The inset shows the proposed schematic model for prevented formation of the ECS in the X\%Zn@ZIF-67 model system. (b) The OTA spectra of X\%Zn@ZIF-67 at 5 ns time delay following 1000 nm excitation. (c) The comparison of kinetics of ESC absorption at $610 \mathrm{~nm}$ and GSB recovery at $585 \mathrm{~nm}$ (inset).

While the experiments with Zn@ZIF-67 model systems show that the presence of $\mathrm{Zn}^{2+}$ can partially block Co-Co communication (which suggests that metal-to-metal communication is likely responsible for the long-lived ECS state in ZIF-67), it is necessary to provide direct evidence to confirm this mechanism. To do so, we designed a second model system, where open-shell $\mathrm{Cu}^{2+}$ ions are used as guest metals to replace $\mathrm{Co}^{2+}$ centers in ZIF-67 (X\%Cu@ZIF-67). The OTA spectra show that X\%Cu@ZIF-67 samples with variable Cu content can also form a long-lived ECS state (Figure S11), where the lifetime for ECS formation (Figure 3a) and GSB recovery (Figure $3 \mathrm{~b}$ ) remains similar to that of the parent ZIF, suggesting that the presence of redox-active $\mathrm{Cu}^{2+}$ does not inhibit ECS state formation. To evaluate the interaction between Cu and Co in X\%Cu@ZIF-67, we utilized X-ray transient absorption (XTA) spectroscopy, a powerful element-specific time-resolved technique, to directly probe the electron density changes at the $\mathrm{Cu}^{2+}$ centers following selective excitation of the $\mathrm{Co}^{2+}$ centers. XTA experiments at the $\mathrm{Cu}$ K-edge were performed using $590 \mathrm{~nm}$ excitation, where $\mathrm{Co}^{2+} \mathrm{d}-\mathrm{d}$ transitions are at a maximum absorbance and $\mathrm{Cu}^{2+} \mathrm{d}-\mathrm{d}$ transitions have negligible absorption (for tetrahedral ligand fields). Figure $3 \mathrm{c}$ shows the XANES spectrum of 15\%Cu@ZIF-67 at the Cu K-edge, which features a strong white line absorption corresponding to the $\mathrm{Cu} 1 \mathrm{~s}-4 \mathrm{p}$ transition. Also shown in Figure $3 \mathrm{c}$ are the difference spectra obtained after subtracting the ground state XANES spectrum from the spectrum collected at delay times at 120 and 500 ps following laser excitation, which demonstrates the transient signals of $\mathrm{Cu}$ due to excitation of Co. A clear positive feature was observed at $8.99 \mathrm{keV}$, which overlaps with the $1 \mathrm{~s}-4 \mathrm{p}$ transition edge, indicating that the edge energy of Cu shifts to lower energy. Because no transient signal (lower set in Figure $3 \mathrm{c}$ ) was observed in the 17\%Cu@ZIF-8 control sample (inert parent framework with $\mathrm{Zn}^{2+}$ node), we can rule out the possibility that the observed Cu edge shift in 15\%Cu@ZIF-67 is due to direct excitation of $\mathrm{Cu}^{2+}$ centers. As a result, we can conclude that the reduction of $\mathrm{Cu}$ arises from the excitation of $\mathrm{Co}$, unambiguously demonstrating a metal-tometal charge transport mechanism through node-to-node communication in X\%Cu@ZIF-67, although the degree of charge delocalization remains uncertain to us. It is also important to note that while the proposed model resembles an electron hopping mechanism, a band transport mechanism may contribute to charge migration in ZIFs. 


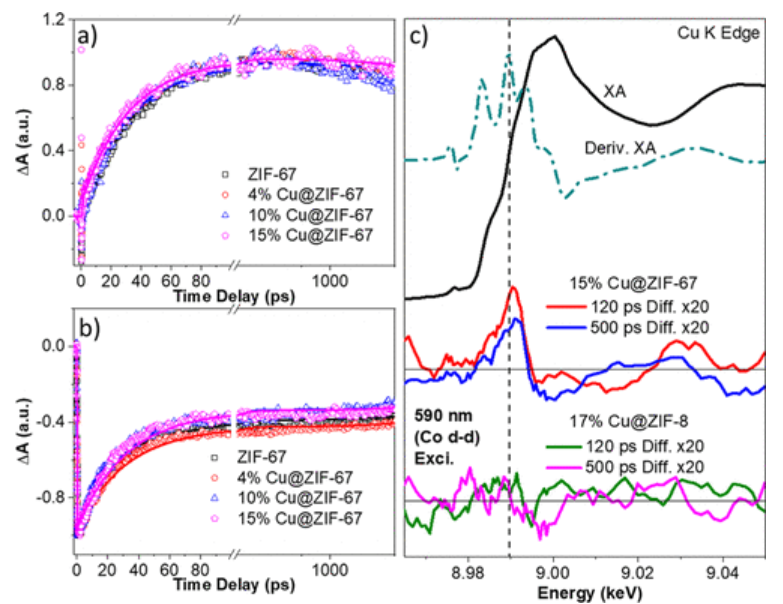

Figure 3. Kinetic traces of the ESC state at $610 \mathrm{~nm}$ (a) and the GSB at $585 \mathrm{~nm}$ (b) for ZIF-67 and Cu@ZIF-67 with variable \% $\mathrm{Cu}$. (c) Representative Cu K-edge XAS spectrum (black, XA) and first derivative spectrum, XTA difference spectra at the Cu Kedge for Cu@ZIF-67 (top set) and Cu@ZIF-8 (bottom set) after 590 nm excitation at 120 and 500 ps time delays.

In summary, we have reported for the first time the charge transport properties of ZIFs using a combination of multiple spectroscopic methods. We designed two mixed-metal ZIFs by incorporating a guest metal $\left(\mathrm{Zn}^{2+}\right.$ or $\mathrm{Cu}^{2+}$ ) to the ZIF-67 parent framework and directly probed the interaction between the guest metals and the metal centers in the parent framework. The paramagnetic susceptibility measurements on both ZIF-67 and Cu@ZIF-67 indicate the presence of intrinsic antiferromagnetic ordering of metal nodes, demonstrating ground-state quantum level communication between $\mathrm{Co}^{2+} / \mathrm{Co}^{2+}$ and $\mathrm{Co}^{2+} / \mathrm{Cu}^{2+}$ nodes. Using optical transient absorption spectroscopy, we found that replacing $\mathrm{Co}^{2+}$ with closed-shell $\mathrm{Zn}^{2+}$ retarded the formation of an ECS state after $\mathrm{Co}^{2+} \mathrm{d}-\mathrm{d}$ excitation, suggesting the presence of $\mathrm{Co}-\mathrm{Co}$ communication in ZIF-67 and charge delocalization within the long-lived ECS state. In contrast, when a redox active metal, $\mathrm{Cu}^{2+}$, is introduced into ZIF67 , we directly observed the migration of charge from photoexcited $\mathrm{Co}^{2+}$ to $\mathrm{Cu}^{2+}$ centers using X-ray transient absorption (XTA) spectroscopy, unambiguously confirming the charge transport pathway through node-to-node communication between different units of ZIFs. These findings not only advance the scientific understanding of the optical and electrical properties of ZIFs, paving the way for their novel application in photoenergy conversion, but also demonstrate an original approach to study porous ZIFs at the molecular level using spectroscopic techniques. Moving forward, computational studies on the excited state electronic structures are necessary to gain further information on the charge transport mechanism in ZIFs.

The authors declare no competing financial interest.

\section{Acknowledgments}

This work was supported by National Science Foundation (DMR-1654140) and ACS-PRF (57503-DNI6). JFB thanks the NSF for support through CHE-1669994. Use of the Advanced Photon Source at Argonne National Laboratory was supported by the U.S. Department of Energy, Office of Science, Office of Basic Energy Sciences, under Award No. DE-AC02-06CH11357.

\section{References}

1 Scholes, G. D.; Fleming, G. R.; Olaya-Castro, A.; van Grondelle, R. Nat. Chem. 2011, 3, 763, DOI: 10.1038/nchem.1145

2 Maza, W. A.; Padilla, R.; Morris, A. J. J. Am. Chem. Soc. 2015, 137, 8161, DOI: 10.1021/jacs.5b03071 
3 Furukawa, H.; Cordova, K. E.; O’Keeffe, M.; Yaghi, O. M. Science 2013, 341, 974

4 Zhang, T.; Lin, W. B. Chem. Soc. Rev. 2014, 43, 5982, DOI: 10.1039/C4CS00103F

5 Wang, J.-L.; Wang, C.; Lin, W. ACS Catal. 2012, 2, 2630, DOI: 10.1021/cs3005874

6 Zhou, H.-C.; Long, J. R.; Yaghi, O. M. Chem. Rev. 2012, 112, 673, DOI: 10.1021/cr300014x

$\underline{7}$ Lu, W.; Wei, Z.; Gu, Z.-Y.; Liu, T.-F.; Park, J.; Park, J.; Tian, J.; Zhang, M.; Zhang, Q.; Gentle lii, T.; Bosch, M.; Zhou, H.-C. Chem. Soc. Rev. 2014, 43, 5561, DOI: 10.1039/C4CS00003J

8 Cohen, S. M. Chem. Rev. 2012, 112, 970, DOI: 10.1021/cr200179u

g-Kent, C. A.; Liu, D.; Meyer, T. J.; Lin, W. J. Am. Chem. Soc. 2012, 134, 3991, DOI: 10.1021/ja211271m

10 Son, H. J.; Jin, S. Y.; Patwardhan, S.; Wezenberg, S. J.; Jeong, N. C.; So, M.; Wilmer, C. E.; Sarjeant, A.

A.; Schatz, G. C.; Snurr, R. Q.; Farha, O. K.; Wiederrecht, G. P.; Hupp, J. T. J. Am. Chem. Soc. 2013, 135, 862, DOI: 10.1021/ja310596a

11 Kent, C. A.; Mehl, B. P.; Ma, L. Q.; Papanikolas, J. M.; Meyer, T. J.; Lin, W. B. J. Am. Chem. Soc. 2010, 132, 12767, DOI: 10.1021/ja102804s

12 Zhang, Z.-M.; Zhang, T.; Wang, C.; Lin, Z.; Long, L.-S.; Lin, W. J. Am. Chem. Soc. 2015, 137, 3197, DOI: 10.1021/jacs.5b00075

13 Maza, W. A.; Morris, A. J. J. Phys. Chem. C 2014, 118, 8803, DOI: 10.1021/jp501140r

14 Yang, S.; Fan, D.; Hu, W.; Pattengale, B.; Liu, C.; Zhang, X.; Huang, J. J. Phys. Chem. C 2018, 122, 3305, DOI: 10.1021/acs.jpcc.8b00471

15 Lee, C. Y.; Farha, O. K.; Hong, B. J.; Sarjeant, A. A.; Nguyen, S. T.; Hupp, J. T. J. Am. Chem. Soc. 2011, 133, 15858, DOI: 10.1021/ja206029a

16 Phan, A.; Doonan, C. J.; Uribe-Romo, F. J.; Knobler, C. B.; O’Keeffe, M.; Yaghi, O. M. Acc. Chem. Res. 2010, 43, 58, DOI: 10.1021/ar900116g

17 Chen, B. L.; Yang, Z. X.; Zhu, Y. Q.; Xia, Y. D. J. Mater. Chem. A 2014, 2, 16811, DOI: 10.1039/C4TA02984D

18 Park, K. S.; Ni, Z.; Côté, A. P.; Choi, J. Y.; Huang, R.; Uribe-Romo, F. J.; Chae, H. K.; O’Keeffe, M.; Yaghi, O. M. Proc. Natl. Acad. Sci. U. S. A. 2006, 103, 10186, DOI: 10.1073/pnas.0602439103

19 Pattengale, B.; Yang, S. Z.; Lee, S.; Huang, J. ACS Catal. 2017, 7, 8446, DOI: 10.1021/acscatal.7b02467

20 Pattengale, B.; Yang, S.; Ludwig, J.; Huang, Z.; Zhang, X.; Huang, J. J. Am. Chem. Soc. 2016, 138, 8072, DOI: 10.1021/jacs.6b04615

$\underline{21}$ Yang, S. Z.; Pattengale, B.; Kovrigin, E. L.; Huang, J. ACS Energy Lett. 2017, 2, 75, DOI: 10.1021/acsenergylett.6b00540

22 Pattengale, B.; Huang, J. Phys. Chem. Chem. Phys. 2018, 20, 14884, DOI: 10.1039/C8CP02078G

23 Butler, K. T.; Hendon, C. H.; Walsh, A. ACS Appl. Mater. Interfaces 2014, 6, 22044, DOI: 10.1021/am507016r

24 Grau-Crespo, R.; Aziz, A.; Collins, A. W.; Crespo-Otero, R.; Hernández, N. C.; Rodriguez-Albelo, L. M.; RuizSalvador, A. R.; Calero, S.; Hamad, S. Angew. Chem., Int. Ed. 2016, 55, 16012, DOI: 10.1002/anie.201609439

25 Butler, K. T.; Hendon, C. H.; Walsh, A. J. Am. Chem. Soc. 2014, 136, 2703, DOI: 10.1021/ja4110073

26 Sun, L.; Campbell, M. G.; Dincă, M. Angew. Chem., Int. Ed. 2016, 55, 3566, DOI: 10.1002/anie.201506219

27 Butler, K. T.; Worrall, S. D.; Molloy, C. D.; Hendon, C. H.; Attfield, M. P.; Dryfe, R. A. W.; Walsh, A. J. Mater. Chem. C 2017, 5, 7726, DOI: 10.1039/C7TC03150E

28 Fei, H. H.; Cahill, J. F.; Prather, K. A.; Cohen, S. M. Inorg. Chem. 2013, 52, 4011, DOI: 10.1021/ic400048g

29 Ban, Y. J.; Li, Y. S.; Peng, Y.; Jin, H.; Jiao, W. M.; Liu, X. L.; Yang, W. S. Chem. - Eur. J. 2014, 20, 11402, DOI: 10.1002/chem.201402287

30 Schejn, A.; Aboulaich, A.; Balan, L.; Falk, V.; Lalevee, J.; Medjahdi, G.; Aranda, L.; Mozet, K.; Schneider, R. Catal. Sci. Technol. 2015, 5, 1829, DOI: 10.1039/C4CY01505C 
31 Shimizu, I.; Morimoto, Y.; Faltermeier, D.; Kerscher, M.; Paria, S.; Abe, T.; Sugimoto, H.; Fujieda, N.; Asano, K.; Suzuki, T.; Comba, P.; Itoh, S. Inorg. Chem. 2017, 56, 9634, DOI: 10.1021/acs.inorgchem.7b01154

32 Casella, L.; Carugo, O.; Gullotti, M.; Doldi, S.; Frassoni, M. Inorg. Chem. 1996, 35, 1101, DOI: 10.1021/ic950392o

33 Solomon, E. I. Inorg. Chem. 2006, 45, 8012, DOI: 10.1021/ic060450d

34 Hoffmann, S. K.; Goslar, J.; Lijewski, S.; Zalewska, A. J. Magn. Reson. 2013, 236, 7, DOI: 10.1016/j.jmr.2013.08.009

35 Carlin, R. L. Magnetochemistry; Springer-Verlag: Berlin, New York, 1986.

\section{Supporting Information}

The Supporting Information is available free of charge on the ACS Publications website at DOI: $10.1021 /$ jacs.8b06727.

Experimental details, structural and optical characterization data, and supplementary SQUID, XAS, and OTA figures $(\underline{P D F})$

pdf

ja8b06727 si 001.pdf (1.75 MB) 